\title{
Innerbetriebliche Ecopreneure durch Umweltmanagementsysteme? - Eine Langzeitanalyse von Bedingungen für proaktives Umweltschutzverhalten
}

\section{Kerstin Pichel}

Online veröffentlicht: 31.10 .2008

(C) Springer-Verlag 2008

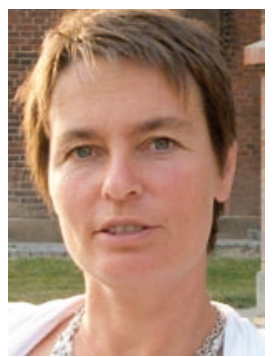

Zusammenfassung Die vorliegende Arbeit thematisiert, welche Unterstützungspotentiale Umweltmanagementsysteme (UMS) für Innerbetriebliches Ecopreneurship - proaktives, umweltbewusstes Arbeitsverhalten aller Unternehmensmitglieder - bieten. Eine Längsschnittuntersuchung in sechs deutschen Unternehmen ermittelt situative und personelle Faktoren, die Ecopreneurship maßgeblich beeinflussen. Unternehmen mit UMS-Aktivitäten weisen positivere Einschätzungen in den relevanten Verhaltensbedingungen und dem Ecopreneurship auf, aber keine kontinuierliche Verbesserungen von Ecopreneurship im Zeitverlauf. Daraus ergeben sich weitere Forschungsfragen, etwa zur Volatilität des proaktiven Arbeitsverhaltens.

Schlüsselwörter proaktiv · Umweltschutzverhalten · Umweltmanagementsysteme · Längsschnittanalyse · Verhaltensbedingungen

Dr. Kerstin Pichel $(\bowtie)$

School of Management and Law,

Stadthausstrasse 14, 8400 Winterthur, Schweiz

E-Mail: Kerstin.pichel@zhaw.ch

\section{Einleitung}

Ecopreneurship - hier verstanden als individuelles, innovatives Umweltschutzengagement von MitarbeiterInnen im Unternehmen - ist eine wesentliche Basis für erfolgreichen betrieblichen Umweltschutz. ${ }^{1}$ Gleichzeitig handelt es sich um ein Extrarollenverhalten, das sich nicht aus Stellenbeschreibungen oder Leistungsvorgaben ableitet, sondern aus persönlicher Verhaltensbereitschaft. ${ }^{2}$ Damit kann das erwünschte Verhalten nicht gefordert sondern muss vornehmlich gefördert werden. Umweltpsychologie und BWL identifizieren eine Vielzahl situativer, individueller und kultureller Faktoren um Ecopreneurship zu fördern. ${ }^{3}$ Umweltmanagementsysteme (UMS) bergen Potentiale die Vielzahl möglicher Verhaltensbedingen systematisch aufbauen und managen zu können und somit eine Förderung umweltbewussten Extrarollenverhaltens zu erzielen. ${ }^{4}$

Und tatsächlich berichten VertreterInnen aus Unternehmen mit UMS von positiven Verhaltensbeeinflussungen: ${ }^{5}$

Femira: „Die Mitarbeiter kommen mehr mit Vorschlägen aus sich heraus. Sie fühlen sich besser informiert und sind interessiert."

Kambium: „Jeder war interessiert, die Vorschläge, die er oder der Kollege gemacht hatte, auch umzusetzen."

Um zu untersuchen, ob diese Anfangseuphorie berechtigt ist, führte ich eine Längsschnittanalyse durch, in der ich den Einfluss von UMS auf relevante Verhaltensbedingun-

\footnotetext{
${ }^{1}$ Kolluru 1994; Ruis-Quintanilla et al. 1996.

${ }^{2}$ Ramus und Steger 2000.

${ }^{3}$ siehe z. B. Hoffman $200536 \mathrm{ff}$; Bansal und Roth 2000; Cordano und Frieze 2000; Hostager et al. 1998; Ramus und Steger 2000; Prakash 2001.

${ }^{4}$ Höppner et al. 1998 74; Hamschmidt und Dyllick 1999 43; UNI und ASU 1997 A34.

${ }^{5}$ Liedtke et al. 199743.
} 
gen und Ecopreneurship selbst untersuchte. Ich analysierte Bedingungen und Verhalten in fünf Unternehmen mit UMS und einem Unternehmen ohne UMS. Die drei Hauptfragen meiner Studie waren:

1. Welche situativen und personellen Faktoren sind wesentliche Bedingungen von Ecopreneurship?

2. Beeinflusst ein UMS diese Ecopreneurship-Bedingungen?

3. Beeinflusst ein UMS das Ecopreneurship-Verhalten?

Nachfolgend skizziere ich Definition und Hypothesen zu Ecopreneurship und seinen Bedingungen sowie zu Einflusspotentialen von UMS. Ich beschreibe die UntersuchungsStichprobe und die quantitative Methode meiner empirischen Untersuchung. Schliesslich präsentiere ich meine Ergebnisse zu den wesentlichen Verhaltensbedingungen und dem Einfluss von UMS. Diese Ergebnisse reflektiere ich vor den Erkenntnissgrenzen der Studie und den Empfehlungen für weitere wissenschaftliche Arbeit zum Thema sowie dem Zusammenspiel der Verhaltensbedingungen.

\section{Untersuchungskonstrukte und Hypothesen}

Ecopreneurship im Verständnis der vorliegenden Untersuchung beschreibt ein formell nicht gefordertes, individuelles, freiwilliges Verhalten von Unternehmensmitgliedern zur Leistungsverbesserung im betrieblichen Umweltschutz. ${ }^{6}$ Es beinhaltet Verhaltensweisen wie die Entwicklung von Verbesserungsvorschlägen, das Nutzen oder Schaffen ökologischer Handlungsmöglichkeiten - beispielsweise den Einbau von Regulierungsmöglichkeiten für die Wasserzufuhr am Arbeitsplatz -, sich informieren über Entwicklungen und Veränderungsmöglichkeiten im betrieblichen Umweltschutz und die Kontaktaufnahme zu anderen Unternehmensmitgliedern um Umweltschutzaktivitäten voran zu bringen. Diese Definition lehnt sich an jene von Petersen (2000) an, weitet aber die betrachteten Akteure über die Geschäftsleitung und Unternehmensgründer hinaus auf alle Unternehmensmitglieder aus.

Bisher konnte sich kein Modell etablieren, um Ecopreneurship zu erklären. ${ }^{7}$ Gemäss der umfassenden Liste potentieller Verhaltensbedingungen ${ }^{8}$ untersuche ich in einer quantitativen Analyse situative und personelle Verhaltensbedingungen. ${ }^{9}$

\footnotetext{
${ }^{6}$ für den Ursprungsbegriff siehe Petersen 2000.

${ }^{7}$ Ramus und Killmer 2005102.

${ }^{8}$ für eine Übersicht siehe Kollmuss und Agyeman 2002.

${ }^{9}$ Zur umfassenden Erklärung von Ecopreneurship untersuchte ich auch Faktoren der Unternehmenskultur (Veränderungsbereitschaft, Wertschätzung von Eigeninitiative, strategische Bedeutung des betrieblichen Umweltschutzes, Promotorenrolle des Umweltschutzbeauftragten). Sie erwiesen sich als offensichtlich verhaltensrelevant, werden aber durch UMS kaum beeinflusst und darum hier nicht weiter thematisiert.
}

Situative Verhaltensbedingungen scheinen massgeblich für die Akzeptanz und Realisierung ökologischen Verhaltens. ${ }^{10}$ Abgeleitet aus verschiedenen organisations- und umweltpsychologischen Forschungsansätzen untersuche ich als situative Verhaltensbedingungen physikalische Ressourcen, Organisationsklima und Schulungen. Die einzelnen Untersuchungskonstrukte sind in Abbildung 1 dargestellt. ${ }^{11}$

Personelle Verhaltensbedingungen wie die Erfolgserwartung, subjektive Umweltschutzseinstellungen und Kontrollüberzeugung gelten ebenfalls als relevant für die Bereitschaft zu ökologischem Arbeitsengagement. ${ }^{12}$ Ich leite aus umwelt- und sozialpsyschologischer Forschung die Untersuchungskonstrukte ökospezifische und ökounspezifische Motivation ab (siehe Abbildung 1).

Die Analyse bisheriger Forschung ergab für nahezu alle Verhaltensbedingungen positive Einflüsse aufUmweltschutzengagement oder eigeninitiatives Arbeitsverhalten. Lediglich extrinsische Arbeitsmotivation und Anreize werden in negativen Zusammenhang mit Ecopreneurship gebracht. ${ }^{13}$ Somit lautet die Hypothese zu den Verhaltensbedingungen:

Hypothese 1: Fast alle situativen und individuellen Verhaltensbedingungen sind signifikant positiv mit Ecopreneurship verbunden, nur Anreize und extrinsische Arbeitsmotivation hängen signifikant negativ mit Ecopreneurship zusammen.

$U M S$, wie das European Eco-Management und Audit Scheme (EMAS) und der International Standard for Environmental Management Systems (ISO 14001) fordern eine systematische und kontinuierliche Entwicklung von Umweltschutzstrategie, -politik und -programmen. Beide Standards heben dabei die Rolle der Mitarbeitenden hervor, ${ }^{14}$ was sich mit dem ressourcenbasierten Strategie-Ansatz deckt. Dieser geht davon aus, dass erfolgreiches Umweltmanagement von internen Fähigkeiten wie Wissen und Verhalten abhängt und diese wiederum fördert. ${ }^{15}$ Auch in der Umweltmanagementpraxis finden sich vielfältige Hinweise auf eine gegenseitige Beeinflussung von Mitarbeitermotivation und Öko-Innovationen. ${ }^{16}$

Die Beeinflussungspotentiale von UMS liegen in ihrer expliziten Forderung der in Abbildung 1 zusammengestellten Verhaltensbedingungen für Ecopreneurship, beispielsweise:

- Bereitstellung geeigneten Arbeitsmaterials. ${ }^{17}$

\footnotetext{
${ }^{10}$ Sharma et al. 1999; Wall 1995; Theyel 2000; Ramus und Steger 2000.

${ }^{11}$ für eine ausführliche Darstellung von Inhalten, Quellen und Thesen zu den Konstrukten vgl. Pichel 2002, 59 ff.

${ }^{12}$ Cordano und Frienze 2000; Taylor und Todd 1995; Guagano et al. 1995; Hines et al. 1986; Hostager et al. 1998; Kastenholz 1994; Luyben und Cummings 1981/1982.

${ }^{13}$ vgl. Luyben und Cummings 1981/1982; Sharma 2000692.

${ }^{14}$ ISO 14001 1996, A. 4. 1; EMAS II 2001, Annex I, B4.

${ }^{15}$ Bansal 2005; Hart 1995; Sharma und Vredenburg 1998.

${ }^{16}$ UNC-ELI 2001; Rennings et al. 2003; Rondinelli und Vastag 2000.

${ }^{17}$ EMAS II 2001, Annex I, A. 4. 1; ISO 14001 1996, 3.5, 4.3 .4 b, 4.4.1.
} 
Abb. 1 Untersuchungskonstrukte und Hypothesen zur Förderung von Ecopreneurship

\section{Management Verhaltensbedingungen Verhalten System}

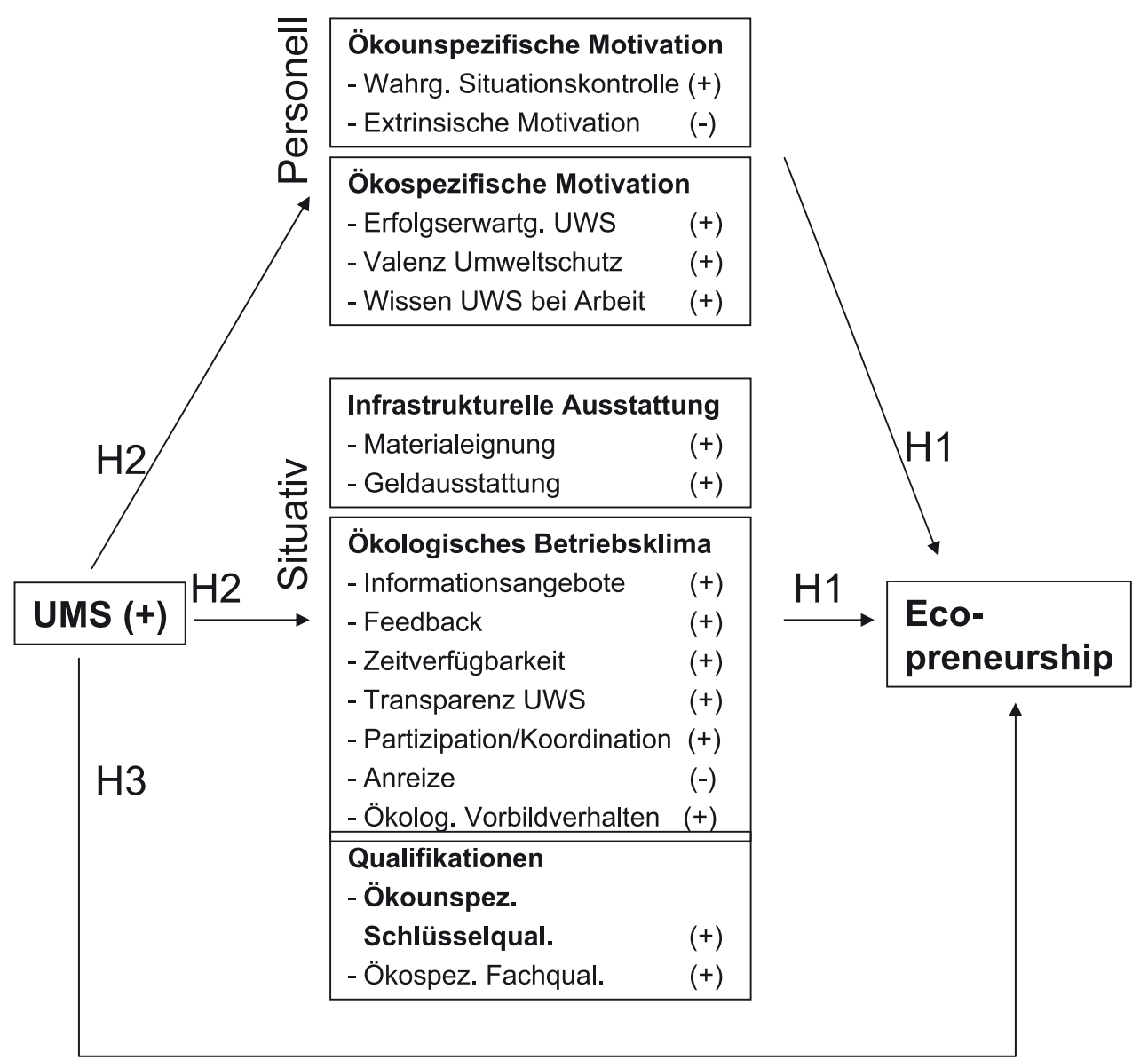

- Bereitstellung von Informationen zu Umweltschutzaktivitäten im Unternehmen. ${ }^{18}$

- Bereitstellung von Möglichkeiten zur Partizipation und Koordination. ${ }^{19}$

- Angebot von Trainings und Förderung des Umweltbewusstseins. ${ }^{20}$

Somit ist die Hypothese vertretbar, dass UMS situative und personelle Ecopreneurship-Bedingungen, die proaktives Verhalten fördern, positiv beeinflussen (siehe Abbildung 1).

Hypothese 2a: Die Einschätzung der meisten situativen und personellen Verhaltensbedingungen ${ }^{21}$ ist signifikant besser je länger ein Unternehmen mit einem UMS arbeitet.

Hypothese 2b: Währen der zwei Untersuchungsjahre verbessert sich die Einschätzung der meisten situativen und

${ }^{18}$ EMAS II 2001, Annex I, A. 4. 1; ISO 14001 1996, 4.4.5c.

${ }^{19}$ EMAS II 2001, Article 1.2.d, Annex I, B. 4. 1; ISO 14001 1996, 3.5, 4.3.4 b, 4.4.1.

${ }^{20}$ EMAS II 2001, Annex I, A. 4. 2; ISO 14001 1996, 4.4.2.

${ }^{21}$ mit Ausnahme der extrinsischen Arbeitsmotivation. personellen Verhaltensbedingungen ${ }^{22}$ ist signifikant in jenen Unternehmen, die mit einem UMS arbeiten, nicht aber im Unternehmen ohne UMS.

Ausgehend von obiger Annahme, dass situative und personelle Faktoren Ecopreneurship zu einem grossen Teil prägen, folgt zu dem die letzte Hypothese:

Hypothese 3a: Ecopreneurship wird in Unternehmen mit UMS als signifikant ausgeprägter angegeben als im Unternehmen ohne UMS.

Hypothese 3b: Während der zwei Untersuchungsjahre verbessert sich das Ecopreneurship signifikant in den Unternehmen mit UMS, nicht aber im Unternehmen ohne UMS.

\section{Stichprobe und Untersuchungsmethodik}

Zur Untersuchung des Einflusses von UMS auf Ecopreneurship und seine Bedingungen, führte ich eine zweijährige

${ }^{22}$ mit Ausnahme der extrinsischen Arbeitsmotivation. 
Langzeitanalyse in sechs deutschen Industrieunternehmen durch. Zwei der Untersuchungsunternehmen arbeiteten bereits seit ca. zwei Jahren mit einem UMS: ein kleiner Tofu-Hersteller (30 Mitarbeitende) und eine mittelgrosse Fahnendruckerei (90 Mitarbeitende). Während des Untersuchungszeitraums führten drei weitere Unternehmen ein UMS ein: ein kleines Galvanik-Unternehmen (40 Mitarbeitende), ein mittelgrosser Betrieb der Kunststoffverarbeitung (190 Mitarbeitende) und ein grosses Metallverarbeitendes Unternehmen (500 Mitarbeitende). Ein Lampenhersteller (360 Mitarbeitende) unternahm keinerlei UMS-Aktivitäten und diente als Kontrollstandort.

Die unterschiedliche Branchenzusammensetzung der Unternehmen ermöglichte eine Untersuchung verschiedenartiger Schwerpunkte innerhalb der UMS-Aktivitäten und somit eine möglichst breite Abdeckung der potentiellen Verhaltensbedingungen.

Für die quantitative Analyse situativer und personeller Verhaltensbedingungen führte ich zwischen 1997 und 1999 drei Befragungsrunden mit Fragebögen in den sechs Unternehmen durch. Die Teinahme war freiwillig. In den kleineren Unternehmen lud ich alle Mitarbeitenden aller Hierarchieebenen zur Teilnahme ein; in den grösseren Unternehmen bildete ich Stichproben aus 50-70 Mitarbeitenden unterschiedlicher Hierarchieebenen und Abteilungen, deren Zusammensetzung ich zugunsten der Vergleichbarkeit möglichst in jeder Befragungsrunde replizierte. In der ersten Befragungsrunde nahmen 231 Mitarbeitende teil, in der zweiten 239 und in der dritten 251. Da ich die Fragebögen persönlich verteilte und wieder einsammelte lag die Rücklaufquote zwischen $68 \%$ und $100 \%$.

Zur Operationalisierung der Untersuchungskonstrukte führte ich explorative Interviews mit drei UMS-Beratern, drei UMS-Auditoren, sechs Umweltschutzbeauftragten und drei UMS-ForscherInnen durch. Für die Itemformulierung stützte ich mich soweit wie möglich auf existierende, bewährte Fragebögen zum proaktiven umweltbewussten Arbeitsverhalten. ${ }^{23}$ Der Fragebogen enthielt hauptsächlich geschlossene Fragen zur Einschätzung der Verhaltensbedingungen (6 stufige Likert-Skala) und zur Beschreibung des Ecopreneurship (4 stufige Likert-Skala). Um eine möglichst wahrheitsgetreue Verhaltensdarstellung zur erreichen, liess ich die quantitativen Angaben zu eigenen Umweltschutzaktivitäten durch Beispiele ergänzen: Wie oft und welche Vorschläge hat der/die Befragte zum Betrieblichen Umweltschutz gemacht, welche Veränderungen beim eigenen Arbeitsverhalten zugunsten des Umweltschutzes wurden realisiert, wie oft hat sich der/ die Befragte zum Betrieblichen Umweltschutz informiert und zu diesem Thema Kontakt mit relevanten KollegInnen aufgenommen?

${ }^{23}$ für eine ausführliche Darstellung vgl. Pichel, 2002, 59ff.
Zur Skalenentwicklung nutzte ich eine explorative principle component Analyse mit Varimaxrotation (Eigenwert $>1$ ). Cronbachs Alpha als Reliabilitätskoeffizient der ermittelten Skalen lag für jeden der drei Erhebungsdurchläufe meist zwischen 0,6 und 0,9 .

Gemäss der Survey-Feedback Methode,$^{24}$ habe ich nach jeder Erhebungsrunde die Ergebnisse Mitarbeitenden und Vorgesetzten jedes Unternehmens vorgestellt und meine Interpretationen der beobachtbaren Veränderungen diskutiert.

\section{Untersuchungsergebnisse}

Folgende drei Teilergebnisse geben die Forschungsresultate wieder: Bedeutung situativer und personeller Verhaltensbedingungen für Ecopreneurship, Beeinflussung von Ecopreneurship und seiner Bedingungen durch UMS und Veränderungen von Ecopreneurship und seiner Bedingungen im Längsschnitt.

Situative und personale Bedingungen von Ecopreneurship

Zur Ermittlung der Bedeutung situativer und personaler Verhaltensbedingungen für Ecopreneurship, führte ich Korrelations- und Regressionsanalysen durch. Als Stichprobe dienten alle Befragten aus Erhebungsrunde drei $(n=251)$ sowie die unidentischen Datensätze der grossen Unternehmen aus Erhebungsrunde zwei ( $\mathrm{n}$ unidentisch $=121$, total $\mathrm{n}=372$ ).

Die Korrelationsanalyse zeigt für nahezu alle Verhaltensbedingungen positive Zusammenhänge mit Ecopreneurship. ${ }^{25}$ Lediglich die Variablen der Infrastrukturellen Ausstattung und die Anreize korrelieren nicht signifikant mit dem Verhalten. Die extrinsische Arbeitsmotivation zeigt den erwarteten negativen Zusammenhang mit dem proaktiven Umweltschutzverhalten.

Da es zwischen den Untersuchungskonstrukten deutliche Interkorrelationen gibt, führte ich eine multiplen Regressionsanalyse durch, die überlagernde Erklärungseffekte bereinigt.

Unter der Verwendung aller 16 Verhaltensbedingungen leisten in der multiplen Regressionsgleichung fünf Verhaltensbedingungen einen signifikanten Beitrag zur Erklärung der Unterschiede im aktiven umweltbewussten Arbeitsverhalten (vgl. Tabelle 1). Durch diese fünf signifikanten Bedingungsvariablen können rund 31,5\% der Varianz des erfragten Ecopreneurships aufgeklärt werden. Der Prüfwert für den gesamten Test (F-Wert) beträgt 27,372 und bestätigt signifikant die Zusammenhänge der Regressionsbeziehung $(\mathrm{p}<0,001)$. Nachfolgend stelle ich die fünf erklärenden

\footnotetext{
${ }^{24}$ Bowers 1973 .

${ }^{25}$ vgl. Pichel, 2002, 142.
} 
Tabelle 1 Kennwerte der regressionsanalytisch ermittelten Prädiktoren für aktives umweltbewusstes Arbeitsverhalten

\begin{tabular}{lcccl}
\hline $\begin{array}{l}\text { Prädiktoren der schrittweisen, multiple Regressions- } \\
\text { analyse (N = 372) }\end{array}$ & $\boldsymbol{\beta}$ & $\mathbf{T}$ & Sig. T & $\mathbf{R}^{\mathbf{2}}$ \\
\hline Transparenz betr. Umweltschutzaktivitäten &, 305 & 5,063 &, 000 &, 207 \\
Ökospez. Fachqualifikation &, 248 & 4,618 &, 000 &, 254 \\
Wahrgenommene Situationskontrolle &, 190 & 3,668 &, 000 &, 287 \\
Valenz betriebl. Umweltschutz &, 143 & 2,957 &, 003 &, 306 \\
Anreize &,- 105 & $-2,039$ &, 042 &, 315 \\
\hline
\end{tabular}

Verhaltensbedingungen und ihre Vorhersagekraft im Einzelnen vor.

Transparenz von Umweltschutzaktivitäten bei der Arbeit ( $\beta=.30$ ) erklärt $20 \%$ der Verhaltensvarianz. Transparenz vermittelt eine Orientierung über die betrieblichen Umweltschutzaktivitäten und eine Einschätzung des eigenen Beitrags zur gesamtbetrieblichen Umweltschutzanstrengung. Vor dem Hintergrund des für Umweltschutzaktivitäten typischen Gefangenendilemmas. ${ }^{26}$ ist die individuelle Einschätzbarkeit der Beiträge beteiligter Akteure erfolgsrelevant, da sonst Zweifel über die Erfolgsaussichten und Anschlussfähigkeit eigener Anstrengungen Handlungsinitiative verhindern können.

Ökologiespezifische Fachqualifikationen $(\beta=, 24)$ erklären weitere 5\% der Varianz von Ecopreneurship. Die Qualifikationen vermitteln Kompetenzen für Umweltschutzaktivitäten bei der Arbeit. Interessanterweise korreliert die Qualifikationsvariable vergleichsweise gering mit der Variable „Wissen über Umweltschutzaufgaben am Arbeitsplatz“ $\left(, 150^{* *}\right)$ aber höher mit der Variable „Transparenz betrieblicher Umweltschutzaktiväten“ (,438**). Diese Interkorrelationen deuten darauf hin, dass Qualifikationen vor allem Klarheit über Sinn und Gesamteinbettung des zu erlernenden Verhaltens vermitteln.

Die wahrgenommene Situationskontrolle $(\beta=, 19)$ erklärt weitere $3 \%$ der Verhaltensvarianz und ist als einzige Verhaltensbedingung nicht ökologiespezifisch. Sie beschreibt das Selbstbewusstsein in die eigenen Einflussmöglichkeiten und effektiven Verhaltensweisen. Unternehmensmitglieder, die überzeugt von ihrer eigenen Entschlusskraft und Umsetzungskompetenz sind, zeigen aktiveres Umweltschutzverhalten am Arbeitsplatz als solche mit geringer Situationskontrolle. $^{27}$

Valenz des Umweltschutzes $(\beta=, 14)$ erklärt überraschenderweise nur weitere 2\% der Varianz von Ecopreneurship. Das ökologische Problembewusstsein mit den Aspekten der persönlichen Betroffenheit und wahrgenommenen Ernsthaftigkeit hat weniger Einfluss auf individuelle Umweltschutzinitiativen bei der Arbeit als die Transparenz über laufende Umweltschutzaktivitäten im Betrieb.

${ }^{26}$ Endres 1985 13ff.

${ }^{27}$ vgl. Campell und Pritchard 1976 85-91; Hostager et al. 1998.
Anreize sind die die letzte erklärende Verhaltensbedingung $(\beta=-, 10)$ welche noch ein zusätzliches Prozent der Verhaltensvarianz erklären. Der Zusammenhang zwischen Anreizen und Ecopreneurship ist negativ und bestätigt die Ergebnisse einer Befragung von 181 ManagerInnen der kanadischen Öl- und Gasindustrie. ${ }^{28}$ Neben der inhaltlichen Erklärung, dass Anreize ein Exekutivdenken gegenüber dem erwünschten Verhalten erzeugen und somit die innovative Eigeninitiative verringern können ${ }^{29}$ gibt es auch eine statistische Erklärungsmöglichkeit: den Suppressionseffekt. ${ }^{30} \mathrm{Da}$ Anreize nur in Kombination mit den Variablen der Qualifizierung zu einem signifikanten Verhaltensprädiktor werden, ist es wahrscheinlich, dass Anreize und Qualifikationen einen gemeinsamen Aspekt haben, der ihre Varianz bestimmt. $\mathrm{Zu}$ denken ist etwa an eine ökologieorientierte Verhaltenserwartung oder -forderung seitens der Geschäftsleitung, die sich sowohl in der Vergabe von Anreizen für das gewünschte Verhalten widerspiegelt als auch in der Lancierung von Qualifizierungen zur persönlichen Ermöglichung des gewünschten, ökologischen Arbeitsverhaltens. Dieser fordernde Aspekt überlagert möglicherweise einen anderen, verhaltensrelevanten Varianzaspekt der Qualifizierungen, etwa die persönliche Befähigung oder Orientierung. Eine solche Prädiktorbeeinträchtigung wird durch eine Suppressionsvariable „,bereinigt“

Diese Ergebnisse der quantitativen Analyse situativer und personeller Verhaltensbedingungen bestätigen Hypothese 1 weitgehend: Nahezu alle quantitativ untersuchten Verhaltensbedingungen weisen positive und signifikante Korrelationen mit Ecopreneurship auf. Aus vier der fünf Bedingungsdimensionen identifiziert die Regressionsanalyse eine Variable als verhaltensrelevant. Nur die Bedingungsdimension der Ressourcen weist keine signifikanten Korrelationen und Regressionen auf. Die extrinsische Motivation hängt signifkant negativ mit Ecopreneurship zusammen, Anreize haben keine signifikante Korrelation und scheinen in der Regressionsanalyse eine Suppressionsbedeutung zu haben.

\footnotetext{
${ }^{28}$ Sharma 2000692.

${ }^{29}$ vgl. Diekmann und Preisendörfer 1991; Schahn 1993, 40; Luyben und Cummings 1981/1982, Diensberg 1997, $111 \mathrm{ff}$., Wulff 1994, Simon 1993, Kohn 1993.

${ }^{30} \mathrm{vgl}$. Bortz 1999, $442 \mathrm{ff}$.

${ }^{31}$ Bortz 1999, 445.
} 
Abb. 2 UMS-spezifische Mittelwertunterschiede für Befragung 3

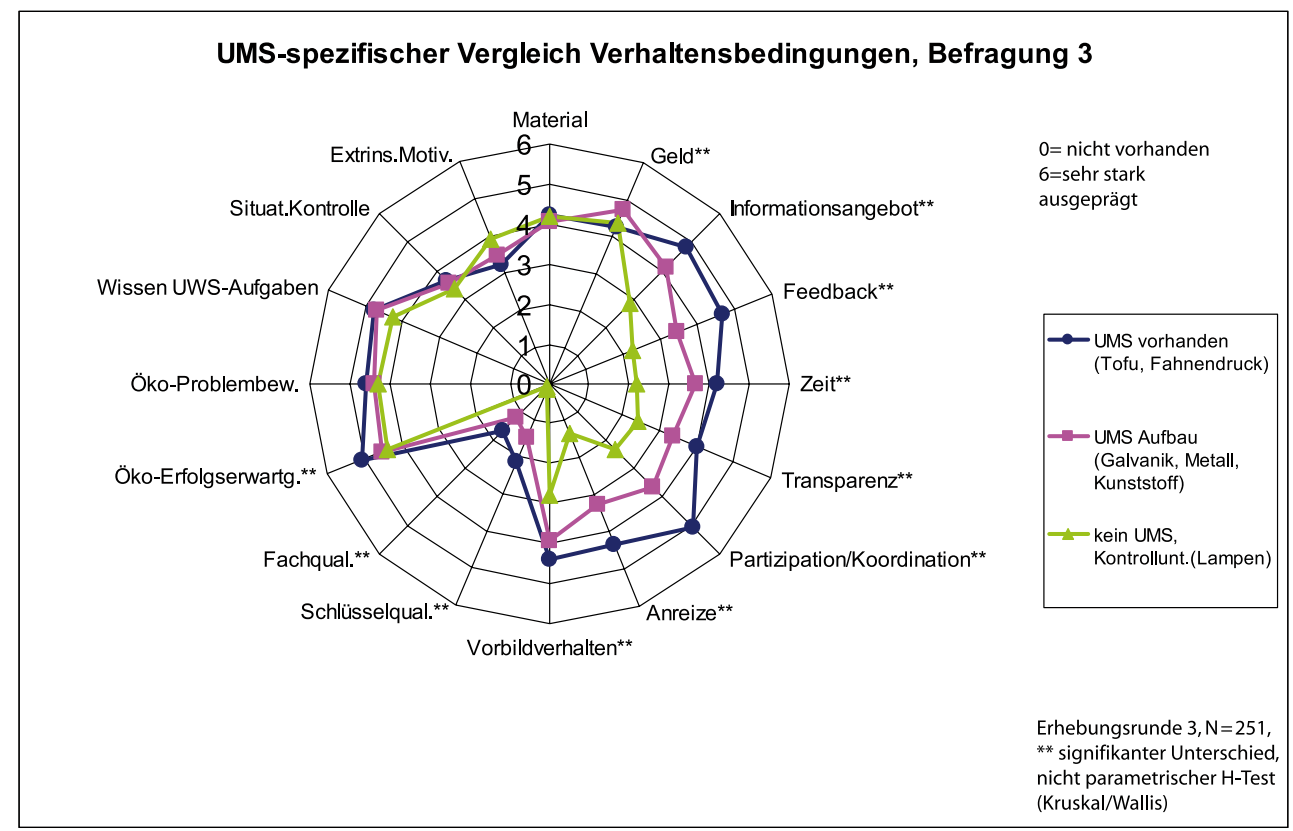

Übergreifend entsteht der Eindruck, dass orientierungsgebende Verhaltensbedingungen positiv und kontrollbezogene Bedingungen negativ mit dem Ecopreneurship zusammen hängen.

\section{Einfluss von UMS}

Zur Untersuchung des Einflusses von UMS auf Verhaltensbedingungen und Ecopreneurship habe ich die Stichproben geclustert nach Unternehmen, bei denen bereits vor meiner Untersuchung eine UMS vorhanden war (Tofu, Fahnendruckerei), solchen, die während der Längsschnittanalyse ein UMS aufbauten (Galvanik, Metallverarbeitung, Kunststoffverarbeitung) und dem Kontrollstandort ohne jegliche UMS-Aktivitäten (Lampenhersteller). Ich verglich die quantitativ ermittelten Einschätzungen zu Verhaltensbedingung und Ecopreneurship in diesen Clustern mit Hilfe eines nichtparametrischen H-Test. ${ }^{32}$

In allen drei Erhebungsrunden bewerteten die Befragten die Verhaltensbedingungen der Dimensionen Betriebsklima und Qualifikationen signifikant besser, je länger mit einem UMS gearbeitet wurde (vgl. beispielhaft Abbildung 2).

Interessanterweise variieren personelle Verhaltensbedingungen - ökospezifische und -unspezifische Motivation nicht in Abhängigkeit von der Dauer des UMS. Wahrscheinlich werde diese individuellen Bedingungen weniger von einem neuen Managementsystem beeinflusst als situative Bedingungen.

Die wichtigsten Verhaltensbedingungen werden umso besser eingeschätzt, je länger ein UMS vorhanden ist:

\footnotetext{
${ }^{32}$ Kruskal und Wallis, Bortz 1999.
}

Transparenz und Fachqualifikationen. Die weniger wichtigen Verhaltensbedingungen - ökologisches Problembewusstsein und Situationskontrolle - variieren nicht gemäss UMS-Dauer.

Das gleiche Bild ergibt sich für Ecopreneurship. Der statistsische $\mathrm{H}-\mathrm{Test}^{33}$ eigt, dass in jeder der drei Erhebungsrunden das Verhalten in Unternehmen mit vorhandenem UMS signifikant aktiver war, als in Unternehmen, die ein UMS aufbauten. Und in den Unternehmen, die ein UMS aufbauten war Ecopreneurship signifikant stärker ausgeprägt als im Kontrollbetrieb.

Hypothesen 2a und 3a können somit zum grossen Teil als bestätigt gelten: Situative Verhaltensbedingungen und Ecopreneurship werden in Unternehmen mit vorhanden UMS besser bewertet, als in jenen, die ein UMS aufbauen und dort besser als im Kontrollstandort.

\section{Entwicklungen im Längsschnitt}

Die Hypothesen $2 \mathrm{~b}$ und $3 \mathrm{~b}$ prognostizieren eine Verbesserung der meisten Verhaltensbedingungen und des Ecopreneurship im Untersuchungsverlauf in jenen Unternehmen, die ein UMS haben, nicht aber im Kontrollstandort. Ich verglich die Entwicklung von Verhaltensbedingungen und Ecopreneurship über die drei Erhebungszeitpunkte mit Hilfe eines nichtparametrischen. ${ }^{34}$

Die Veränderungen am Kontrollstandort zeigen keine systematische, signifikante, erklärbare Entwicklung. In den Experimentalstandorten verbesserte sich die Einschät-

\footnotetext{
${ }^{33}$ Kruskal und Wallis, Bortz 1999 z.

${ }^{34}$ U-Test Mann und Whitney, Bortz 1999, vgl. Tabelle 2.
} 
Tabelle 2 Mittelwertvergleiche zwischen Befragung 1 und 3

\begin{tabular}{|c|c|c|c|c|c|c|c|}
\hline $\begin{array}{l}\text { Unter- } \\
\text { suchungs- } \\
\text { dimensionen }\end{array}$ & $\begin{array}{c}\text { Bedingungen: } \\
\text { 1= sehr gut; } \\
6=\text { sehr schlecht; } \\
\text { Verhalten: je po- } \\
\text { sitiver Z-Werte, } \\
\text { um so aktiver }\end{array}$ & $\begin{array}{c}\text { Mittelwerte } \\
\text { Befragg. 1, } \\
\text { N = 167 } \\
\text { Experimental- } \\
\text { standorte: Tofu, } \\
\text { Galvanik, Me- } \\
\text { tall, Kunststoff }\end{array}$ & $\begin{array}{c}\text { Mittelwerte } \\
\text { Befragg. 3, } \\
\text { N =169 } \\
\text { Experimental- } \\
\text { standorte: Tofu, } \\
\text { Galvanik, Me- } \\
\text { tall, Kunststoff }\end{array}$ & $\begin{array}{c}\text { Signifikanz der } \\
\text { Mittelwert- } \\
\text { unterschiede } \\
\text { (nichtparame- } \\
\text { trischer U-Test } \\
\text { nach Mann und } \\
\text { Whitney) }\end{array}$ & $\begin{array}{l}\text { Mittelwerte } \\
\text { Befragg. 1, } \\
\mathrm{N}=49 \\
\text { Kontrollstand- } \\
\text { ort: Lampen- } \\
\text { hersteller }\end{array}$ & $\begin{array}{l}\text { Mittelwerte } \\
\text { Befragg. 3, } \\
\mathbf{N}=49 \\
\text { Kontrollstand- } \\
\text { ort: Lampen- } \\
\text { hersteller }\end{array}$ & $\begin{array}{c}\text { Signifikanz der } \\
\text { Mittelwert- } \\
\text { unterschiede } \\
\text { (nichtparame- } \\
\text { trischer U-Test } \\
\text { nach Mann und } \\
\text { Whitney) }\end{array}$ \\
\hline \multirow[t]{2}{*}{ Ressourcen } & Material & 3,07 & 2,92 & n.s. & 3,36 & 2,82 & n.s. \\
\hline & Geld & 2,45 & 2,30 & n.s. & 2,42 & 2,63 & n.s. \\
\hline Ökologisches & Infoangebot & 3,23 & 2,76 &, 001 & 4,21 & 4,13 & n.s. \\
\hline Betriebs- und & Feedback & 3,21 & 3,39 & n.s. & 4,98 & 4,75 & n.s. \\
\hline \multirow[t]{5}{*}{ Arbeitsklima } & Zeit & 3,79 & 3,28 & ,003 & 4,52 & 4,81 & n.s. \\
\hline & Transparenz & 3,93 & 3,51 & ,004 & 4,66 & 4,57 & n.s. \\
\hline & Partizipation & 3,47 & 3,11 & 022 & 4,77 & 4,69 & n.s. \\
\hline & Anreize & 3,76 & 3,64 & n.s. & 5,60 & 5,67 & n.s. \\
\hline & Vorbild & 3,20 & 2,95 & n.s. & 4,32 & 4,21 & n.s. \\
\hline \multirow{2}{*}{$\begin{array}{l}\text { Qualifika- } \\
\text { tionen }\end{array}$} & Schlüsselqualif. & 5,35 & 5,40 & n.s. & 6,74 & 6,86 & n.s. \\
\hline & Fachqualif. & 5,70 & 5,71 & n.s. & 6,87 & 6,89 & n.s. \\
\hline $\begin{array}{l}\text { Ökospezi- } \\
\text { fische }\end{array}$ & Ökoerfolg & 2,31 & 2,36 & n.s. & 2,51 & 2,62 & n.s. \\
\hline \multirow[t]{2}{*}{ Motivation } & Ökovalenz & 2,40 & 2,56 & n.s. & 2,80 & 2,70 & n.s. \\
\hline & Wissen & Nicht erfasst & 2,29 & Nicht erfasst & Nicht erfasst & 2,72 & Nicht erfasst \\
\hline Ökounspez. & Situat. Kontrolle & 3,33 & 3,39 & n.s. & 3,65 & 3,66 & n.s. \\
\hline Motivation & $\begin{array}{l}\text { Extrinsische } \\
\text { Motiv. }\end{array}$ & 4,04 & 3,79 & n.s. & 3,87 & 3,27 &, $049 *$ \\
\hline $\begin{array}{l}\text { Ecopreneur- } \\
\text { ship, z-Werte }\end{array}$ & &, 098 &, 079 &, 046 &,- 25 &,- 28 & n.s. \\
\hline
\end{tabular}

zung der meisten situativen Verhaltensbedingungen. Als signifikant werden im nichtparametrischen U-Test nach Mann und Whitney die Verbesserungen des Informationsangebots, der Zeitausstattung, der Transparenz von Umweltschutzaktivitäten bei der Arbeit, der Partizipation/ Koordination ermittelt - also der wesentlichen Verhaltensprädiktoren. Die auffälligen aber nicht signifikanten Veränderungen der anderen Verhaltensbedingungen beruhen meist auf Entwicklungen in einzelnen Unternehmen, die nicht repräsentativ für die Gesamtheit der Stichprobe sind. Interessanterweise gibt es keine signifikanten Veränderungen in der personellen Verhaltensbedingungen, wahrscheinlich erneut ein Zeichen, dass individuelle Faktoren durch ein UMS weniger leicht oder schnell beeinflussbar sind.

Trotz der signifikanten Verbesserungen der verhaltensrelevanten situativen Bedingungen verschlechtert sich das Ecopreneurship von Befragung $1 \mathrm{zu}$ Befragung 3 signifikant (siehe Tabelle 2). In den Ergebnispräsentationen und -diskussionen nannten die Befragten als Grund hierfür, dass eine Reihe von Umweltschutzmaßnahmen nun angestossen sei und sie nicht ständig neue Ideen liefern können.

Das weniger aktiv angegebene umweltbewusste Arbeitsverhalten kann auch als eine Art konstruktive Unzufrieden- heit ${ }^{35}$ gedeutet werden: die ersten Umweltaktivitäten am Arbeitsplatz wurden in Befragung 1 möglicherweise noch als besondere Vorschläge oder Optimierungsmaßnahmen wahrgenommen und im Fragebogen angegeben, während sie in der dritten Befragungsrunde zum alltäglichen, veränderten Arbeitsverhalten geworden waren. $\mathrm{Zu}$ denken ist etwa an einen genaueren Tofuzuschnitt oder eine konsequentere Reinigung der Galvanisierungsgegenstände, um Ausschuß zu vermeiden.

Angesichts dieser Ergebnisse können Hypothesen $2 b$ und 3 b nur ansatzweise bestätigt werden. Die durch ein UMS angestossenen Veränderungen der Verhaltensbedingungen sind offenbar und plausiblerweise eng verzahnt mit anderen gesamtbetrieblichen Veränderungen und gestalten sich darum unternehmensspezifisch. Das proaktive Umweltschutzverhalten bei der Arbeit ist wahrscheinlich gekennzeichnet durch eine gewisse Volatilität: aussergewöhnliches Engagement kann nicht kontinuierlich realisiert werden, sondern zeigt sich wellenförmig. Nach besonderen Aktivitäten braucht es eine gewisse Zeit der Etablierung und Generierung neuer Ideen. Diese Etablierung aussergewöhnlicher Verhaltensweisen führt wahrscheinlich auch zu einer

\footnotetext{
${ }^{35}$ vgl. Bruggemann 1974.
} 
Gewöhnung an die veränderten Abläufe, so dass sie nicht mehr als aussergewöhnlich wahrgenommen werden.

\section{Fazit und Schlussfolgerung: wollen wir wirklich Ecopereneure?}

In dieser Studie wurden als wichtigste verhaltensrelevante personelle Bedingungen von Ecopreneurship das ökologische Problembewusstsein und die wahrgenommene Situationskontrolle ermittelt. Als wichtigste situative Bedingungen stellten sich die Transparenz betrieblicher Umweltschutzaktivitäten und Fachqualifikationen heraus. Anreize hängen negativ mit Ecopreneurship zusammen, was wahrscheinlich durch den statistischen Suppressionseffekt bedingt ist und die Bedeutung eines selbstbestimmten Betriebsklimas hervorheben mag.

Tatsächlich führt die längere Etabliertheit eines UMS zu besserer Beurteilung situativer Verhaltensbedingungen und Ecopreneurship.

Nichts desto trotz bleibt festzustellen, dass während des Untersuchungsverlaufs unternehmensspezifische Entwicklungen die Verhaltensbedingungen stark beeinflussten und das untersuchte Umweltschutzverhalten am Arbeitsplatz nicht kontinuierlich aktiver wurde. UMS heben die unternehmensspezifischen Strömungen, Volatilitäten von aussergewöhnlichem Engagement und mögliche Reaktionsverzögerungen in der Umsetzung veränderter Verhaltensbedingungen offensichtlich nicht völlig auf.

Vor allem die Ecopreneurshipentwicklung lässt weitere Forschung interessant erscheinen. So scheint es wünschenswert, kongruente Definitionen und Operationalisierungen von Verhalten und Verhaltensbedingungen zu wählen, um Ergebnisse genauer miteinander vergleichen zu können. Die Verhaltensdefinition und Operationalisierung dieser Untersuchung erwies sich als empirie-tauglich, die Konstruktbildung zum Betriebsklima sollte überprüft werden. Für eine weitere Erhebung sollte mit einer grösseren Stichprobe von Unternehmen gearbeitet werden, um betriebsspezifische Entwicklungen aus der Einflussanalyse kompensieren zu können. Ausserdem scheint eine längere Analysezeit sinnvoll, um die Entwicklung des Verhaltens besser erklären zu können.

Abschliessend möchte ich auf eine grundsätzliche Frage aufmerksam machen, die sich auf Grund der Ergebnisse stellt. Offensichtlich ist ein forderndes, kontrollierendes Betriebsklima kontraproduktiv für ein selbstbestimmtes, proaktives Umweltschutzverhalten am Arbeitsplatz. Stattdessen scheinen eher Faktoren, die Autonomie und Einblicke in gesamtbetriebliche Aktivitäten ausdrücken, Ecopreneurship zu fördern. UnternehmensvertreterInnen sollten sich vor der öffentlichen Forderung nach dem erwünschten Umweltschutzverhalten der Unternehmensmitglieder überlegen, ob Ecopreneurship auf breiter Linie tatsächlich zur Unterneh- menskultur passt und realistischerweise von der Mehrzahl der Unternehmensmitglieder getragen werden kann. Trägt das Führungsverhalten und das individuelle Leistungsverständnis ein eigenverantwortliches Handeln der meisten MitarbeiterInnen? Möglicherweise ist eine differenzierte Definition der umweltbezogenen Rollen je Hierarchieebene unternehmensadäquater ${ }^{36}$.

\section{Autorbiographie}

Frau Dr. Pichel beschäftigt sich als Dozentin für Strategisches Management an der Zürcher Hochschule für angewandte Wissenschaft seit über 10 Jahren mit der Förderung eigenverantwortlichen, nachhaltigen Handelns im Betrieb. Ihre Beratungserfahrungen bei der Einführung von Umweltmanagementsystemen (UMS) mündeten in der Langzeitanalyse der Verhaltensauswirkungen von UMS.

\section{Literatur}

Bansal P (2005) Evolving sustainability: A longitudinal study on corporate sustainable development. Strategic Management Journal $26: 197-218$

Bird A (1996) Training for Environmental Improvement. In: Wehrmeyer W (Hrsg) Greening people, Human resources und environmental management. Greenleaf Publishing, Sheffield

Bortz J (1999) Statistik für Sozialwissenschaflter, 5. Auflage. Springer, Berlin

Campell JP, Pritchard RD (1976) Motivation theory in industrial und organisational psychology. In: Dunette MD (Hrsg) Handbook of industrial und organisational psychology. Rund McNally, Chicago, S 63-130

Cordano M, Frieze IH (2000) Pollution reduction preferences of US environmental managers: applying Ajzens's theory of planned behaviour. Academy of Management Journal 43(4):627-641

Bowers DG (1973) OD Techniques und Their Results in 23 Organizations: The Michigan ICL Study. The Journal of Applied Behavioral Science 9(1):21-43

Diekmann A, Preisendörfer P (1991) Umweltbewusstsein, ökonomische Anreize und Umweltverhalten - Empirische Befunde aus der Berner und Münchner Umweltbefragung. Schweizerische Zeitschrift für Soziologie 2:207-231

Diekmann A, Preisendörfer P (1992) Persönliches Umweltverhalten. Diskrepanz zwischen Anspruch und Wirklichkeit. Kölner Zeitschrift für Soziologie und Sozialpsychologie 44:226-251

EMAS II (2001) Verordnung (EG) Nr. 761/2001 EMAS II des Europäischen Parlaments und Rates vom 27.4.01, über die freiwillige Beteiligung von Organisationen an einem Gemeinschaftssystem für das Umweltmanagement und die Umweltbetriebsprüfung. Amtsblatt der Europäischen Gemeinschaft. Brüssel

Endres A (1985) Umwelt- und Ressourcenökonomie. Wissenschaftliche Buchgesellschaft, Darmstadt

Floyd SW, Lane PW (2000) Strategizing through the organisation: Managing role conflict in strategic renewal. Academy of Management Review 25(1):154-177

Frese M (1985) Stress at work und psychosomatic complaints: a causal interpretation. Journal of applied psychology 70:314-328

Guagano GA, Stern PC, Dietz T (1995) Influences on attitude-behavior relationship: A natural experiment with curbside recycling. Environment und Behavior 5:699-718

\footnotetext{
${ }^{36}$ vgl. Floyd und Lane 2000.
} 
Hamschmidt J, Dyllick T (1999) ISO 14001 in der Praxis; Wirkungen von Umweltmanagementsystemen in Schweizer Unternehmen. IWÖ-Diskussionsbeitrag Nr. 75. IWÖ-HSG, St. Gallen

Hart SL (1995) A natural-resource-based view of the firm. Academy of Management Review 20:986-1014

Hines JM, Hungerford HR, Tomeran AA (1986) Analysis und Synthesis of Research on Responsible Environmental Behavior: A MetaAnalysis. Journal of Environmental Education 2:1-8

Hoffman AJ (2006) Getting ahead of the Curve: Corporate Strategies that adress Climate Change. University of Michigan

Hoffman AJ (2005) Prepare for the Pew Center on Global Climate Change. University of Michigan

Höppner N-O, Sietz M, Seuring S, Czaja F (1998) Öko-Audit-Verordnung - Ergebnisse einer Befragung validierter Unternehmen. UWF 2:73-76

Hostager TJ, Neil TC, Decker RL, Lorentz RD (1998) Seeing environmental opportunities: effects of intrapreneurial ability, efficacy, motivation und desirability. Journal of Organizational Change Management II(1):11-25

ISO 14001 (1996) Umweltmanagementsysteme, Spezifikation mit Anleitung zur Anwendung. Berlin

Kastenholz HG (1994) Bedingungen umweltverantwortlichen Handelns in einer Schweizer Bergregion: eine empirische Studie unter der besonderen Berücksichtigung anthropogen verursachter Klimaveränderungen. P. Lang, Bern

Kohn A (1993) Punished by rewards: the trouble with gold stars, incentive plans, A's, praise und other bribes. Houghton Mifflin, New York

Kollmuss A, Agyeman J (2002) Mind the gap: why do people act environmentally und what are the barriers to pro-environmental behavior? Environmental Education Research 8(3):239-260

Kolluru RV (1994) Environmental Strategies Handbook. McGrawHill, Inc., New York

Liedtke C, Jürgens G, Rohn H (1997) Zukunftsfähiges Unternehmen (2) - Beurteilung des Öko-Audits im Hinblick auf Ressourcenmanagement in kleinen und mittleren Unternehmen. Wuppertal Inst. für Klima, Umwelt, Energie, Wuppertal (Wuppertal Papers, 72)

Luyben PD, Cummings S (1981/1982) Motivation Beverage Container Recycling on a College Campus. Journal of Environmental Systems 11:235-245

Peters LH, O'Connor EJ (1980) Situational Constraints und Work Outcomes: The Influences of a Frequently Overlooked Construct. The Academy of Management Review 5(3):391-397

Petersen H (2000) Die bewegten Gründer. Ökologisches Wirtschaften 2:4-5

Pichel K (2002) Innerbetriebliches Ecopreneurship durch Umweltmanagementsysteme? Dissertation TU Berlin, http://deposit.ddb.de/ cgi-bin/dokserv?idn=970378505

Prakash A (2001) Why do firms adapt „beyond-compliance“ environmental policies? Business Strategy und the Environment 10:286-299

Ramus CA, Killmer AB (2005) Corporate greening through Prosocial Extrarole Behaviours - A conceptual Framework for Employee motivation. Online article in advance of print in Business Strategy und the Environment, http://www3.interscience.wiley.com/ cgi-bin/abstract/112126905/ABSTRACT/, found $28^{\text {th }}$ November 2005

Ramus CA, Steger U (2000) The roles of supervisory support behaviors und environmental policy in employee ,ecoinitiatives“ at leading-edge European companies. Academy of Management Journal 47(4):605-626

Rennings K, Ziegler A, Ankele K, Hoffmann E, Nill J (2003) The Influence of the EU Environmental Management und Auditing Scheme on Environmental Innovations und Competitiveness in Germany: An Analysis on the Basis of Case Studies und a LargeScale Survey. Discussion Paper No. 03-14. Zentrum für Europäische Wirtschaftsforschung $\mathrm{GmbH}$, Mannheim

Rondinelli DA, Vastag G (2000) Panacea common sense or just a label? The value of ISO 14001 environmental management system. European Management Journal 18(5):499-510

Ruiz-Quintanilla A, Bunge J, Freeman-Gallant A, Cohen-Rosenthal E (1996) Employee participation in pollution reduction: a sociotechnical perspective. Business Strategy und the Environment 5(3):137-144

Schahn J (1993) Psychologische Maßnahmen zur Mülltrennung und -vermeidung. In: Schahn J, Giesinger T (Hrsg) Psychologie für den Umweltschutz. Belzt Psychologie Verlags Union, Weinheim, S. $123-132$

Sharma S (2000) Managerial interpretations und organizational context as predictors of corporate choice of environmental strategy. Academy of Management Journal 43(4):681-697

Sharma S, Pablo A, Vredenburg H (1999) Corporate Environmental Responsiveness Strategies: The Importance of Issue Interpretation und Organizational Context. Journal of Applied Behavioral Science 35(1):87-108

Sharma S, Vredenburg H (1998) Proactive corporate environmental strategy und the development of competitively valuable organizational capabilities. Strategic Management Journal 19:729-753

Taylor S, Todd P (1995) Understanding household garbage reduction behaviour: a test of an integrated model. Journal of Public Policy und Marketing 14(2):192-205

Theyel G (2000) Management practices for environmental innovation und performance. International Journal of Operations \& Production Management 20(2):249-266

UNC-ELI, University of North Carolina und Environmental Law Institute (2001) Drivers, designs und consequences of environmental management system. Environmental Law Institute, Washington D.C.

UNI/ASU (1997) Öko-Audit in der mittelständischen Praxis, Evaluierung und Ansätze für eine Effizienzsteigerung von Umweltmanagementsystemen in der Praxis. Unternehmerinstitut e. V./ Arbeitsgemeinschaft Selbständiger Unternehmer, Bonn

Wall S (1995) Barriers to Individual Environmental Action: The Influence of Attitudes und Social Experiences. The Canadian Review of Sociology und Anthropology 32:465-489

Weiner B (1986) An attributional theory of motivation und emotion. Springer, New York 